\title{
Mix Breed
}

National Cancer Institute

\section{Source}

National Cancer Institute. Mix Breed. NCI Thesaurus. Code C53951.

A dog that is not purebred. 